Bryn Mawr College

Scholarship, Research, and Creative Work at Bryn Mawr

College

1976

\title{
Proton spin relaxation in dilute methane gas: A symmetrized theory and its experimental verification
}

Peter A. Beckmann

Bryn Mawr College, pbeckman@brynmawr.edu

M. Bloom

I. Ozier

Let us know how access to this document benefits you.

Follow this and additional works at: https://repository.brynmawr.edu/physics_pubs

Part of the Physics Commons

\section{Custom Citation}

Beckmann, Peter A., M. Bloom, and I. Ozier. 1976. "Proton spin relaxation in dilute methane gas: A symmetrized theory and its experimental verification." Canadian Journal of Physics 54.16: 1712-1727.

This paper is posted at Scholarship, Research, and Creative Work at Bryn Mawr College. https://repository.brynmawr.edu/physics_pubs/121

For more information, please contact repository@brynmawr.edu. 


\title{
Proton spin relaxation in dilute methane gas: A symmetrized theory and its experimental verification $^{1}$
}

\author{
P.A. Beckmann, ${ }^{2,3}$ M. Bloom, and I. Ozier ${ }^{4}$ \\ Canadian Journal of Physics, 1976, 54(16): 1712-1727 https://doi.org/10.1139/p76-204
}

\begin{abstract}
Nuclear spin relaxation in low density methane gas is investigated theoretically and experimentally. A theory is developed in which full account is taken of the tetrahedral symmetry of the molecule. Fora nuclear Larmor frequency of $30 \mathrm{MHz}$. the time evolution of the nonequilibrium magnetization is measured as a function of density between approximately 0.005 and 17 amagats at temperatures of 110,150 , and $295 \mathrm{~K}$. In all cases, exponential relaxation is observed. By using the theory in conjunction with the known spin rotation constants and rotational energy levels of $\mathrm{CH}_{4}$, the measured values of the relaxation rate $R_{l}$, have been fit very well at each temperature, both for the maximum value of $R_{l}$ which contains no adjustable parameters and for the density dependence of $R_{l}$, which contains a single parameter taken to be the collision cross section for molecular reorientation. The centrifugal distortion splittings of the rotational levels are shown to have an important influence on the observed values of $R_{l}$ at 30 MHzand, more generally on the dependence of the time evolution of the nonequilibrium magnetization on density and frequency. On the basis of the theory, a new type of 'relaxation rate spectroscopy' is proposed. Nonexponential relaxation is predicted to occur at tow densities when the nuclear Larmor frequency is tuned to a centrifugal distortion splitting.
\end{abstract}

\section{Introduction}

The methane molecule provides a rich field for study by the methods of nuclear spin relaxation. Two areas are of particular interest in the current work. First, there is the opportunity to study nuclear spin conversion caused by molecular collisions. Secondly, the energy splitting for certain pairs of rotational energy levels involved in the relaxation process is comparable with nuclear Larmor frequencies and with collision frequencies that can be studied experimentally. The investigation of these two topics forms a major part of the present paper.

Nuclear spin relaxation in low density molecular gases results from the nuclear hyperfine interactions which couple the nuclear spins to the molecular rotation. (Yi et al. 1968; Bloom 1972). In their study of the relaxation rate $\mathrm{R} 1$ in $\mathrm{CH} 4$ as a function of density and temperature, Bloom et al. (1967) showed that the dominant relaxation mechanism in methane is the intramolecular spin rotation interaction. By going to lower densities at room temperature, Dong and Bloom (1970) were able to observe the characteristic maximum in the density dependence of $R_{l}$ and were able to interpret their results successfully without taking into account the splitting of the $J$ levels by centrifugal distortion effects. In a more detailed room temperature study with improved techniques over a wider range in density, Beckmann et al. (1972) showed that

\footnotetext{
${ }^{1}$ Research supported by the National Research Council of Canada.

${ }^{2}$ Present address: Department of Physics, University of Nottingham, Nottingham NG7 2RD, England.

${ }^{3}$ National Research Council of Canada Predoctoral Fellow, 1972-1975.

${ }^{4}$ Alfred P. Sloan Research Fellow, 1972-1974.
} 
transitions between those levels with the same $J$ but different distortion energies make a significant contribution to $R_{l}$. These effects were introduced into their analysis in a phenomenological way.

In the current work, the theory of spin relaxation in $\mathrm{CH}_{4}$ is extended to take full account of the permutation symmetry of the four protons. The centrifugal distortion splitting, the correlation between the rotation and spin quantum numbers, and the fully symmetrized spinrotational wave functions are treated in detail. This theoretical calculation of the time evolution of the magnetization after perturbation from its equilibrium value is presented in Sect. 2. In order- to test the theory, measurements have been made of this time evolution at temperatures of $110 \mathrm{~K}, 150 \mathrm{~K}$, and $295 \mathrm{~K}$ as a function of density between 0.005 and 17 amagats. The experiments were done at a frequency of $30 \mathrm{MHz}$ using conventional pulsed techniques. In each case, exponential relaxation was observed to within experimental accuracy. In Sect. 3, the experimental methods are discussed and the measurements of $R_{l}$ are presented. In Sect. 4, the experimental results are compared with theory. At each temperature, the agreement is very good, both for the maximum value of $R_{l}$, which involves no adjustable parameters, and for the shape of the density dependence, which is determined by a single parameter taken to be the collision cross-section for molecular reorientation. In Sect. 5, the conclusions are summarized, the current theory is compared to earlier treatments, and the major limitations of the approach adopted here are pointed out.

Although the present paper is intended to be self-contained, the complexity has forced us to omit many of the details. For a more complete discussion, the reader is referred to Beckmann (1975).

An interesting extension of the current experimental method is proposed based on the calculated time evolution of the magnetization. It is predicted that, if the relaxation rate were measured at a fixed low density as a function of frequency, then relaxation anomalies will occur when the Larmor frequency is close to a centrifugal distortion splitting between levels coupled by the spin rotation interaction. The relaxation anomaly should be characterized by nonexponential relaxation. Its observation would establish a new type of spectroscopy. This 'relaxation rate spectroscopy' is discussed in Sect. 2.C.

There are several closely related studies of current interest which have provided some of our motivation for establishing a more basic theory of nuclear spin relaxation in dilute $\mathrm{CH}_{4}$ gas:

(A) Methane is the prototype tetrahedral molecule; the results obtained here may be. useful in extending the work of Courtney and Armstrong (1972) on other spherical tops and in interpreting some of the results on simple symmetric tops (Armstrong and Courtney 1972).

(B) Attempts have been made to observe interstellar methane using the infrared spectrum (Herzberg 1969, 1971) and the microwave spectrum (Broten 1974). ${ }^{5}$ When results are obtained, their interpretation will depend on the relative abundance of different nuclear spin species and hence on an understanding of nuclear spin relaxation.

(C) Unsuccessful efforts have been made to prepare samples of $\mathrm{CH}_{4}$ gas which deviate significantly from the equilibrium distribution over the different spin species (Curl et al. 1966). While a preliminary explanation for this failure has been given (Curl et al. 1967; Ozier and Yi 1967), the detailed treatment required can be derived on the basis of the current work.

${ }^{5}$ Broten, N. W. 1974. Private communication. 
(D) The problems of neutron scattering by methane gas (Hama and Miyagi 1973) and of nuclear spin conversion in solid methane at low temperatures (Bloom and Morrison 1973; Press and Kollmar 1975) are closely related to the one discussed here.

\section{Theory}

\section{A. The Relaxation Equations}

In a general relaxation experiment, the spin system is initially prepared in a nonequilibrium state in some specified way and the deviation $M(t)$ of the magnetization from the equilibrium value is measured as a function of time. Here in Sect. 2, we shall set up general equations determining this time dependence and solve them for the particular experimental conditions which were used. Since we are interested in the behavior of $M(t)$ at low densities, we shall discuss the problem in terms of transitions between states of the isolated molecule; these transitions are caused by intermolecular collisions.

$$
M(t)=\sum_{I} M^{I}(t)=\sum_{I m} m n_{I m}(t)
$$

where $M^{I}(t)$ is the deviation from equilibrium of the magnetization associated with spin species $I$ and $n_{I m}(t)$ is the deviation from equilibrium of the population of state $(\mathrm{Im})$.

We now make the assumption that the nuclear spin relaxation can be described in terms of rate equations for the $n_{I m}(t)$. In the high temperature approximation, the master equation for the population deviations (Abragam 1961) can be written

$$
\frac{\mathrm{d} n_{I m}}{\mathrm{~d} t}=\sum_{I^{\prime} m^{\prime}} S\left(I m ; I^{\prime} m^{\prime}\right) n_{I^{\prime} m^{\prime}}
$$

Here the off-diagonal coefficients $S\left(\operatorname{Im} ; I^{\prime} m^{\prime}\right)$ are simply the transition rates $W\left(\operatorname{Im} ; I^{\prime} m^{\prime}\right)=$ $W\left(I^{\prime} m^{\prime} ; I m\right)$ between spin states $(I m)$ and $\left(I^{\prime} m^{\prime}\right)$. The diagonal coefficients are given by

$$
S(I m ; I m)=-\sum_{I^{\prime} m^{\prime} \neq I m} W\left(I^{\prime} m^{\prime} ; I m\right)
$$

To each spin state, there correspond many $\cdot$ rotational levels which are labelled by a family of quantum numbers collectively designated as $\Upsilon$. Consequently,

[3] $W\left(I^{\prime} m^{\prime} ; I m\right)=\sum_{\Upsilon^{\prime}} P_{\Upsilon^{\prime}} \sum_{\Upsilon} W\left(\Upsilon^{\prime} I^{\prime} m^{\prime} ; \Upsilon I m\right)$

Here $P r$ is the probability of a molecule being in rotational state $Y$ given that its spin state is $(\mathrm{Im})$. W(YI'm'; $\Re \mathrm{Im})$ is the rate of transitions between the individual. spin-rotational states $(Y$ 'I' $m$ ') and $(Y m)$.

The transitions are produced by the combined effects of the anisotropic intermolecular potential and the intramolecular spin rotation interaction $H_{S R}$. In the absence of the internal magnetic field created by the spin rotation interaction at the site of each proton, the effect of the collisions is to produce transitions between states of different $Y$ but identical (Im) via the anisotropic intermolecular forces. However, the rotational magnetic field $i s$ present and is modulated by the collisions in a random manner, so that transitions between states of different $Y$ and different $(\mathrm{Im})$ can occur provided the initial and final states are coupled by non-zero matrix elements of $H_{S R}$. The transition rate is proportional to the intensity of the power spectrum of the randomly modulated field at the frequency $\omega \gamma^{\prime} I^{\prime} m^{\prime} ; \gamma_{I m}$ corresponding to the energy difference between the states.

It is customary to write (Gordon 1968; Abragam 1961) 


$$
\text { [4] } \begin{aligned}
W\left(\Upsilon^{\prime} I^{\prime} m^{\prime} ; \Upsilon I m\right)=\hbar^{-2} \mid & \left.\left\langle\Upsilon^{\prime} I^{\prime} m^{\prime}\left|H_{\mathrm{SR}}\right| \Upsilon I m\right\rangle\right|^{2} \\
& \times j_{\Upsilon^{\prime} \times}\left(\omega_{\Upsilon^{\prime} I^{\prime} m^{\prime} ; \Upsilon I m}\right)
\end{aligned}
$$

where we have followed Bloom et al. (1967) and introduced the reduced spectral density function $j r^{\prime} r(\omega)$. This is the Fourier transform of the reduced correlation function $g r r(t)$ of $H_{S R}$. When the collision-broadened rotational levels have a Lorentzian form, $g r r(t)$ decays exponentially to 0 from 1 with correlation time $\tau r r$ and

$$
j_{\Upsilon^{\prime} \gamma^{\prime}}(\omega)=2 \tau_{\Upsilon^{\prime} \Upsilon} /\left(1+\omega^{2} \tau_{\Upsilon^{\prime} \Upsilon^{\prime}}\right)
$$

Because the spin rotational field is different at the positions of the four protons, the possibility of spin conversion exists.

\section{B. The Transition Rates}

In order to outline the calculation of the $W\left(I^{\prime} m^{\prime} ; I m\right)$, we must first review briefly the form of the Hamiltonian $H$ and the spin rotational wave-functions $\Psi(\Upsilon \mathrm{Im})$. For an isolated methane molecule in the ground vibronic state,

[6] $H=H_{\mathrm{ROT}}^{\mathrm{s}}+H_{\mathrm{ROT}}{ }^{\mathrm{T}}+H_{\mathrm{Z}}+H_{\mathrm{SR}}^{\mathrm{s}}+H_{\mathrm{SR}}{ }^{\mathrm{T}}$

$H_{R O T}{ }^{S}$ and $H_{R O T}{ }^{T}$ are the scalar and tensor parts, respectively, of the rotational Hamiltonian. Here $H_{R O T}{ }^{S}$ can be taken as the energy of rigid rotation $B_{0} J^{2}$ with $B_{0}=5.241 \mathrm{~cm}^{-1}$ (Tarrago et al.

1975) and $H_{R O T}{ }^{T}$ can be taken as the fourth rank centrifugal distortion term $D_{T} \Omega_{4}$ with $D_{T}=132.9$ $\mathrm{kHz}$ (Holt et a/. 1975). The operator $\Omega_{4}$ and the higher order corrections to both $H_{R O T}{ }^{S}$ and $H_{R O T}{ }^{T}$ are discussed by Ozier (1974). $H_{Z}$ is the Zeeman interaction resulting from the nuclear and rotational magnetic moments. $H_{S R}$ and $H_{S R}{ }^{T}$ are the scalar and tensor parts respectively of $H^{S R}$. The corresponding coupling coefficients are the average spin rotation constant $c_{a}=(10.4 \pm 0.1) \mathrm{kHz}$ and the anisotropy in the spin rotation matrix $c_{d}=(18.5 \pm 0.5) \mathrm{kHz}(\mathrm{Yi}$ et al. 1971).

In the representation in which the first three terms in [6] are diagonal, the individual energy levels are labelled with the quantum numbers $J, m_{J}, \gamma, \rho, t, I$, and $m$. The symbol $\gamma$ introduced earlier stands for the first 5 of the set. The quantities $(\gamma \rho)$ together specify the representation $k$ of the group $T_{d}$ by which the rotational functions $R$ transform: $\gamma=A, E$, or $F$, and $\rho=1$ or 2 . The correspondence between $\mathrm{k}$ and $(\gamma \rho)$ is $A_{l}(A 2) ; A_{2}(A 1) ; E(E 1)$, and $(E 2) ; F_{l}(F l)$; $F_{2}(F 2)$. For given $(J m \jmath \gamma \rho)$, there will be $N(J \gamma \rho)$ distinct rotational levels split by $H_{R O T}{ }^{T}$. These are labelled by $t=1,2, \ldots N(J \gamma \rho)$ in order of increasing eigenvalue of $H_{R O T}{ }^{T}$. The parity of these rotational levels is $p=(-1)^{\mathrm{J}+\rho-1}$. This system of labelling the levels follows the work of Yi et al. (1968) and Fox and Ozier (1970), and uses the definitions of Jahn (1938) for the representations of $T_{d}$. The relationship to other current schemes of labelling these levels is given in Ozier (1974).

The rotational wave functions $R\left(J m_{J} ; \gamma \xi \tau \rho\right)$ for component $\xi$ of representation $(\gamma \rho)$ can be written as a superposition of 'primitive functions' with superposition constants $A_{t K}(J \gamma \xi \tau \rho)$, where $K$ takes specific integral values between 0 and $J$. (See [3] of Ozier and Rosenberg (1973).) These constants were generated by the algorithm of Fox and Ozier (1970); since they diagonalize $H_{R O T}{ }^{T}$, they can in general be determined only numerically. The nuclear spin functions $\chi(\beta \eta ; I m)$ for component $\eta$ of representation $\eta$ are listed in Table V of Yi et al. (1968). ${ }^{6}$ The total wave

${ }^{6}$ The $1 / \sqrt{ } 8$ in $\chi(E 1 ; 00)$ of this table should be $1 / \sqrt{ } 12$. 
functions $\Psi(\eta m)$ are formed by contracting $\chi(\beta \eta ; I m)$ with $R\left(J m_{J} ; \gamma \xi \tau \rho\right)$ on the component indices $\xi$ and $\eta$ (see [41], [54], and [59] of Ozier and Fox (1970)).

The spin irreducible representation $\beta$ is restricted to the values $A_{l}, E$, and $F_{2}$. This leads to a one-to-one correspondence between $I$ and $\beta$, and subsequently to the following correspondence $I \sim \beta \sim \gamma: 2 \sim A_{1} \sim A ; 1 \sim F_{2} \sim F ; 0 \sim E \sim E$. Because levels with different $(\gamma \rho t)$ are split by the centrifugal distortion term $H_{R O T}{ }^{T}$, levels with different (It) are so split as well; any transition which alters $I$ and/or $t$ leads to a change in the distortion energy. The distortion splittings have very important effects on the nuclear spin relaxation process. They range from $10^{7}$ to $10^{10} \mathrm{~Hz}$ for levels which are appreciably populated at room temperature. In Holt et al. (1975), the splitting for $J \leq 21$ are tabulated. In Fig. 1, the splittings for $J=12$ are shown for illustration. Each level has degeneracy $[I+\delta(\gamma, E)](2 I+1)(2 J+1) . H_{Z}$ lifts all the degeneracy except that due to the parity factor $[\mathrm{I}+\delta(\gamma, E)]$.

It is the off-diagonal matrix elements of $H_{S R}$ between pairs of these states $(\gamma \mathrm{Im})$ that drive the transitions involved in the relaxation process (see [4]). The selection rules for the $H_{S R}$ are therefore of primary interest. Because this interaction is linear in the nuclear spins and linear in $J$, general angular momentum theory requires that $\Delta I=0, \pm 1 ; \Delta m=0, \pm 1 ; \Delta J=0, \pm 1 ; \Delta m_{J}=0, \pm$ 1. Because all internal interactions conserve parity, $\Delta \rho=0$ if $\Delta J=0$ and $\Delta \rho \neq 0$ if $\Delta= \pm 1$.

The tetrahedral symmetry imposes additional selection rules. For $H_{S R} S, \Delta I=0, \Delta \gamma=0$, and $\Delta t=0 . H_{S R} S$ thus conserves the distortion energy. Its matrix elements are denoted $\left\langle H_{S R} S(\gamma, \gamma)\right.$ ᄀ. For $H_{S R}{ }^{T}$, the only requirement is that one of the coupled states have $\gamma=F$ and consequently $I=1$. These matrix elements are denoted $\left\langle H_{S R}^{T}\left(\gamma^{\prime}, F\right)\right\rangle$. There is no restriction on $\Delta t$. There are matrix elements diagonal in $I$ only for $I=1$; they vanish for $I=0$ and 2 . There are matrix elements off-diagonal in $I$ and/or $t$ of the type $I=2 \leftrightarrow 1,1 \leftrightarrow 1$, and $0 \leftrightarrow 1 . H_{S R}{ }^{T}$ thus does not conserve $I$ or the distortion energy. The quantum numbers $(I \gamma t)$ are 'good' only because the distortion splittings are much larger than the corresponding off-diagonal elements due to $H_{S R}{ }^{T}$. (The case of Zeeman-tuned avoided crossings is discussed below.)

In, for example [4], we must consider all transitions which satisfy these various selection rules. However, the form of the reduced spectral density function $j r^{\prime} r$ allows us to eliminate the transitions with $\Delta J= \pm 1$. At the low densities of interest here, the collisional broadening of the rotational levels is much less than the separation between levels with different values of $J$, so that $\omega \tau \gamma^{\prime} r$ in [5] is very large for $\Delta J= \pm 1$ and the associated transition rate is negligible.

From the selection rules discussed above, it is clear that for $\Delta J=0$ transitions, the

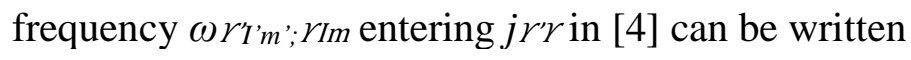

$$
\text { [7] } \begin{aligned}
\omega_{J_{\mathrm{p}}}\left(\gamma^{\prime} t^{\prime} m^{\prime} ; \gamma t m\right)=\omega(J \gamma t \rho)- & -\omega\left(J \gamma^{\prime} t^{\prime} \rho\right) \\
& -\left(m-m^{\prime}\right) \omega_{0}
\end{aligned}
$$

Here $\omega_{0}$ is the difference between the nuclear and rotational Larmor frequencies and $\omega(J \gamma \tau \rho)$ is the eigenvalue of $H_{P O T}^{T}$ in frequency units. Equation 7 gives $\omega_{J \rho}\left(\gamma^{\prime} t^{\prime} m^{\prime} ; \gamma t m\right)$ as the difference between the distortion splitting $\omega r^{\prime} r \equiv \omega(J \gamma \tau \rho)-\omega\left(J \gamma^{\prime} \tau^{\prime} \rho\right)$ and $\left(\mathrm{m}-\mathrm{m}^{\prime}\right) \omega_{0}$. As a function of density, $j r^{\prime} r$ will have a maximum when the collisional broadening of the rotational levels is the order of this difference. At the low densities of interest here, this condition is met for a variety of splittings, with the result that transitions between different distortion levels make an important contribution to the relaxation rate and its dependence on $\mathrm{m} 0$ and density.

Equation 7 simplifies in some cases. When $\Delta I=\Delta t=0$, as occurs for all scalar and some tensor matrix elements, the first two terms cancel and [7] reduces to $-\left(m-m^{\prime}\right) \omega_{0}$. Since completely diagonal matrix elements are excluded from [4], $\Delta m$ must equal \pm 1 . Because the 
frequency enters $j r^{\prime} r$ only as the square, the sign of $\Delta m$ cancels out and $\omega_{J \rho}\left(\gamma^{\prime} t^{\prime} m^{\prime} ; \gamma t m\right)$ reduces to $\omega_{0}$. On the other hand, when $\Delta I$ and/or $\Delta t \neq 0$, as occurs for some tensor matrix elements, the full expression [7] must be used. Here $\Delta m=0$ occurs as well. However, when $\Delta m= \pm 1$, the sign of $\Delta m$ does not cancel, a fact which has important implications for the relaxation problem.

Although each reduced correlation function $g r^{\prime} r$ can have its own correlation time, we will follow Dong and Bloom (1970) and assume that transitions associated with $H_{S R}{ }^{S}$ alone will have correlation time $\tau_{1}$ and those associated with $H_{S R}{ }^{T}$ alone will have correlation time $\tau_{2}$. There will be transitions associated with the cross term (see [11] below) and these will be assumed to have correlation time $\tau_{12}$. The corresponding spectral densities will be denoted $j_{1}, j_{2}$, and $j_{12}$.

The probabilities $P r$, which appear in [3) can, to excellent approximation, be written as a function of $J$ and $\gamma$ only:

$$
P(J, \gamma)=[U(\gamma)]^{-1} \exp \left\{-B_{0} h c J(J+1) / k T\right\}
$$

where

$$
U(\gamma)=\sum_{J} \sum_{0}[2 J+1] N(J \gamma \rho) \exp \left\{-B_{0} h c J(J+1) / k T\right\}
$$

$U(\gamma)$ is not the partition function $Z$ because of the correlation between $\gamma$ and $I$. Rather $U(\gamma)=$ $n_{\gamma} Z / 16$ where $n_{\gamma}$ is the dimensionality of the representation associated with the index $\gamma$.

The matrix elements appearing in [4] have been evaluated with techniques similar to those used by Ozier et al. (1968) to calculate the terms $\left\langle H_{S R}^{T}(F, F)\right\rangle$ for $\Delta t=0$. For use in other applications, the results are listed in the Appendix.

The transition rates can now be evaluated from [3]. By simplifying the sums implicit in [3], each $W\left(I^{\prime} m^{\prime} ; I m\right)$ has been expressed as the product of two factors: the square of a ClebschGordan coefficient containing the nuclear spin quantum numbers and a complex sum over the rotational quantum numbers. The results are:

$$
W\left(2 m^{\prime} ; 2 m\right)=\left[C\left(2,1,2 ; m, m^{\prime}-m, m^{\prime}\right)\right]^{2} \sum_{J \rho} c_{\mathrm{a}}{ }^{2} \widehat{A}(J A \rho)
$$

$$
\begin{gathered}
W\left(1 m^{\prime} ; 1 m\right)=\left[C\left(1,1,1 ; m, m^{\prime}-m, m^{\prime}\right)\right]^{2} \sum_{J_{\rho}} \frac{1}{3} c_{\mathrm{a}}{ }^{2} \hat{A}(J F \rho)+c_{\mathrm{a}} c_{\mathrm{d}} \hat{B}(J F \rho) \\
+c_{\mathrm{d}}{ }^{2} \hat{D}(J F \rho)+c_{\mathrm{d}}{ }^{2} \hat{E}_{m m^{\prime}}(J F \rho) \\
W\left(2 m^{\prime} ; 1 m\right)=\left[C\left(1,1,2 ; m, m^{\prime}-m, m^{\prime}\right)\right]^{2} \sum_{J_{\rho}} c_{\mathrm{d}}{ }^{2} \hat{E}_{m m m^{\prime}}(J A \rho) \\
W(00 ; 1 m)=[C(1,1,0 ; m, 0-m, 0)]^{2} \sum_{J \rho} c_{\mathrm{d}}{ }^{2} \hat{E}_{m 0}(J E \rho)
\end{gathered}
$$

The functions $\hat{A}, \hat{B}, \hat{D}$, and $\hat{E}$ have been introduced as a convenient shorthand

[17] $\hat{E}_{m m^{\prime}}(J \gamma \rho)=\frac{4}{27} \pi^{2} \frac{(2 J+1)}{J(J+1)} P(J \gamma) \sum_{t t^{\prime}}\left[1-\delta(\gamma, F) \delta\left(t^{\prime}, t\right)\right]\left[M_{J \rho}\left(\gamma t^{\prime} ; F t\right)\right]^{2} j_{2}\left\{\omega_{J \rho}\left(\gamma t^{\prime} m^{\prime} ; F t m\right)\right\}$ The Kronecker delta functions in [17] have been inserted to eliminate from $\hat{E}_{m m} \cdot(J \gamma \rho)$ the $(\gamma=F)$ terms diagonal in $t$; these have been included in $\hat{\mathrm{D}}(J \gamma \rho)$. The $M_{j \rho}\left(\gamma t^{\prime} ; F t\right)$ are double sums involving the tetrahedral coefficients $A_{t K}(J \gamma \xi \rho)$. The sums are defined explicitly in the Appendix. 
The different terms in [10] to [13] can be classified according to their values of $\Delta I$ and $\Delta t$. This has been done in Table 1 . The terms in $c_{d}{ }^{2}$ can conserve both, either, or neither $I$ and $t$. The term in $c_{a}{ }^{2}$ and the cross term in $c_{a} c_{d}$ conserve both $I$ and $t$. The effect of this cross term is very small. Unlike $\hat{\mathrm{D}}$ and $\hat{E}$ which involve sums on $M_{j \rho}, \hat{\mathrm{B}}$ involves a sum on $M_{j \rho}$ itself. Although the individual terms are significant, the signs fluctuate. As a result, the sum on $(J t \rho)$ is much smaller than the corresponding sums resulting from $\hat{D}$ and $\hat{E}$. The cross term may be important for individual transitions in particular magnetic fields, but it does not make a significant contribution to the total transition probability $W(\mathrm{~lm} ; \mathrm{lm})$. The term in $c_{a} c_{d}$ will therefore be neglected in the rest of the analysis. This result is of special interest because the cross term is identically zero in the treatment of Dong and Bloom (1970) where the spin symmetry is neglected.

It should be pointed out that relaxation due to the intramolecular dipolar interaction has not been included in the present treatment. It can be shown from the calculations of Bloom et al. (1967) and the discussion of Dong and Bloom (1970) that the contributions of the dipolar interaction to the $W\left(I^{\prime} m^{\prime} ; I m\right)$ are negligible. However, like the $c_{a} c_{d}$ term, this interaction may be important for individual transitions under particular experimental conditions.

\section{C. The Time Dependence of the Magnetization: Relaxation Rate Spectroscopy}

Now that the coefficients $S\left(I m ; I^{\prime} m^{\prime}\right)$ have been determined, the 9 coupled equations, [2], can be solved for $n_{I m}(t)$ for the appropriate initial conditions and [1] can be used to calculate $M(t)$. The solutions are quite complicated and, in general, the relaxation predicted is nonexponential. However, there are many situations in which the relaxation is well approximated by a single exponential with time constant $T_{1}$ and relaxation rate $R_{l}=T_{1}{ }^{-1}$. In particular, all of the experiments reported here can be adequately represented by a single exponential. In this case,

From [1] and [2],

[19]

$$
\mathrm{d} M / \mathrm{d} t \approx-R_{1} M
$$

$$
R_{\mathrm{L}} \approx-\sum_{I m} \sum_{I^{\prime} m^{\prime}} m S\left(I m ; I^{\prime} m^{\prime}\right) n_{I^{\prime} m^{\prime}} / \sum_{I_{m}} m n_{I m}
$$

While [19] can be used to calculate $R_{l}$ when it is experimentally observed that a single rate constant provides a good approximation, it of course provides no information on the form of the correction terms, their physical origin, or the conditions under which nonexponential relaxation can be observed. To investigate this problem further, the 9 coupled equations, [2], were solved by using an iterative procedure in which the $n_{I m}(t)$ are expressed in a Taylor expansion. For iteration $(I+1)$,

$$
n_{I m}[(l+1) \Delta t]=n_{I m}[l \Delta t]+\Delta t \sum_{I^{\prime} m^{\prime}} S\left(I m ; I^{\prime} m^{\prime}\right) n_{I m}[l \Delta t]
$$

By starting with known initial conditions dictated by the experiment, the time evolution of the $n_{I m}$, can be calculated to any desired accuracy by choosing the time interval $\Delta t$ to be sufficiently small. $M(t)$ is then obtained from [1]. In the experiments reported here, the initial conditions correspond to setting the initial deviation $M(0)$ to -2 times the equilibrium magnetization. (As discussed in Sect. 3, the equilibrium magnetization is inverted with a $\pi$ pulse.)

In order to study the degree to which the relaxation is nonexponential, we fit the calculated $M(t)$ to a single exponential for a short interval first near $t=0$ and again near the time $t_{1}$ defined by $M\left(t_{1}\right)=0.2 M(0)$. The resulting relaxation rates $R_{I S}$ and $R_{l L}$ characterize respectively, the short and long time behaviour of the magnetization. In Fig. 2, these two rates are plotted as a function of the nuclear Larmor frequency at a temperature $T$ of $150 \mathrm{~K}$ for $\tau_{1}=\tau_{2}$ $=1.83 \times 10^{-8} \mathrm{~S}$, conditions which correspond to a density of approximately 0.01 amagat. 
It is clear that at low frequencies, including the $30 \mathrm{MHz}$ used here, $R_{I S}$ is very close to $R_{I L}$, so that a single exponential is an excellent approximation and [19] will yield reliable results. However, above about $35 \mathrm{MHz}$, the two rates separate; the difference $\left(R_{l S}-R_{l L}\right)$ shows a maximum in the region of $55 \mathrm{MHz}$ and again in the region of $76 \mathrm{MHz}$.

The interpretation of these maxima points the way to a new type of study which might be called 'relaxation rate spectroscopy'. The origin of these maxima in $R_{l S}-R_{l L}$ lies in the form of the reduced spectral density $j r$ ' $r$ given in [5]. When the external field is adjusted so that ( $m-$ $\left.m^{\prime}\right) \omega_{0}$ equals the splitting between two distortion levels $Y$ and $\Upsilon^{\prime}$ coupled by $H_{S R}, \omega_{J \rho}\left(\gamma^{\prime} t^{\prime} m^{\prime} ; \gamma t m\right)$ $=0$ (see [7]) and the contribution of the corresponding transition goes through a maximum. At lower densities, $t r^{\prime} r$ is longer and the maximum is sharper. At the density used for Fig. 2, the contribution of this 'resonant' transition is large enough to produce marked effects. In fact, this one transition can dominate the contribution of $H_{S R}{ }^{T}$ to the relaxation. The plot shown in Fig. 2 can be interpreted as a 'spectrum' since it reveals the presence of two distortion splittings: $(J=5$, $\gamma=E, \rho=2, t=1) \leftrightarrow(5, F, 2, \mathrm{I})$ at $51.8 \mathrm{MHz}$ and $(3, A, 2,1) \leftrightarrow(3, F, 2, \mathrm{I})$ at $71.7 \mathrm{MHz}$ (Holt et al. 1975). Both of these involve spin conversion, $I=0 \leftrightarrow 1$ and $I=2 \leftrightarrow 1$ respectively, but $I=1 \leftrightarrow 1$ transitions with $\Delta t \neq 0$ have similar effects. The frequency dependence of the relaxation can therefore be used to do a type of spectroscopy.

This occurrence of a 'resonant' transition corresponds to an avoided level crossing such as that used by Ozier et al. (1970) to detect spin conversion with molecular beam methods. The magnetic field tunes $\mathrm{Hz}$ so that the two levels involved become almost degenerate and repel one another. At the avoided crossing, the contribution of these two levels to the relaxation rate is a maximum. For a given field, only states with particular values of $\left(m, m_{J}\right)$ are closely coupled. For example, when level $(\Upsilon, I, m)$ is at an avoided crossing, level $(\Upsilon, I,-m)$ is far from a crossing and makes a much smaller contribution to $R_{1}$. It may be necessary to include the dipolar interaction in a detailed analysis of some individual avoided crossings.

In order to obtain further insight into the time evolution of the magnetization at a nuclear Larmor frequency of $30 \mathrm{MHz}$, an empirical approximation was devised to formulate ${ }^{7}$ the problem in terms of the $M^{I}(t)$ for $\mathrm{I}=1$ and 2. By differentiating [I] and using [2], it can be shown that

$$
\begin{aligned}
& \text { [21] } \frac{\mathrm{d} M^{I}}{\mathrm{~d} t}=\sum_{I^{\prime} m^{\prime}}\left[m^{\prime}+\delta\left(m^{\prime}, 0\right)\right] R^{I}\left(I^{\prime} m^{\prime}\right) n_{I^{\prime} m^{\prime}} \\
& \text { where } \\
& \text { [22] }\left[m^{\prime}+\delta\left(m^{\prime}, 0\right)\right] R^{I}\left(I^{\prime} m^{\prime}\right)= \\
& \sum_{m} m S\left(I m ; I^{\prime} m^{\prime}\right)
\end{aligned}
$$

The $R^{I}\left(I^{\prime} m^{\prime}\right)$ are given in terms of the $W\left(I^{\prime} m^{\prime} ; I m\right)$ in Table 2. It is then useful to introduce the average relaxation function

$$
R^{I I^{\prime}} \equiv-\frac{1}{2 I^{\prime}} \sum_{m^{\prime} \neq 0} R^{I}\left(I^{\prime} m^{\prime}\right)
$$

and to approximate $[21]$ by

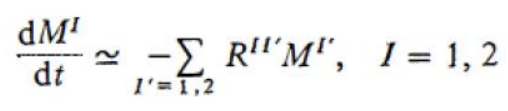

\footnotetext{
${ }^{7}$ Only $M^{(1)}(t)$ and $M^{(2)}(t)$ are of interest because $M^{(0)}(t)=0$. However, as can be seen from [2], the population of the $(I=0)$ species does enter the problem.
} 
The justification for [24] rests on the fact that the results at $30 \mathrm{MHz}$ agree with the iterative solution to within $3 \%$ over the entire range of densities studied.

In order to solve [24], we first write it in matrix form.

$$
\mathrm{d} M / \mathrm{d} t=-R M
$$

There is a matrix $\Lambda^{-1}$ which brings $R$ to diagonal form $\lambda$ through a similarity transformation:

$$
\lambda=\Lambda^{-1} R \Lambda
$$

The matrix $\Lambda$ is not unitary. It is the sum of the components of $M$ that is conserved in the transformation and not the scalar product $M \cdot M$. The columns of $\Lambda, \Lambda^{-1}$ must add to unity. In spite of this, $\Lambda$ is easily calculated because only $2 \times 2$ matrices are involved.

Each of the two eigenvectors of I relaxes exponentially with its own time constant. These two linear combinations of $M^{(1)}$ and $M^{(2)}$ are denoted by $M_{\alpha}$. and $M_{\beta}$ with corresponding relaxation rates $\lambda_{\alpha}$. and $\lambda_{\beta}$. A detailed study of the solution to this problem (Beckmann 1975) shows that at the higher densities, $\lambda_{\alpha}-\lambda_{\beta}$ is significant but $\left|M_{\alpha}\right|$ is negligible relative to $\left|M_{\beta}\right|$. At the lower densities, $\left|M_{\alpha}\right|$ is a significant fraction of $\left|M_{\beta}\right|$ but $\left|\lambda_{\alpha}-\lambda_{\beta}\right|$ is very small. Thus, the prediction is that at all densities a single relaxation rate will be observed within the accuracy of the present experiments: in particular at $30 \mathrm{MHz}$ and in general at any frequency far from all distortion splitting.

The empirical nature of [24] must be emphasized. One sufficient set of conditions that [24] follows directly from [21] without approximation requires that $R^{I}(I, 0)=0$ independent of all $I$ and $I^{\prime}$ and that $R^{I}\left(I^{\prime}, m^{\prime} \neq 0\right)=R_{1} \delta\left(I^{\prime}, I\right)$ independent of all $m^{\prime}$. These conditions also imply exactly exponential relaxation. However, when $I^{\prime} \neq I, R^{I}\left(I^{\prime}, m^{\prime} \neq 0\right)$ is a significant fraction of $R_{I}$ here, so that the second of these conditions is not satisfied. There may be other sets of conditions sufficient to render [24] exact, but until the particular set operative here is found, the application of [24] to other problems must be treated with caution.

\section{Experimental Methods and Results}

The time dependence of the longitudinal nuclear spin relaxation rate was measured at 30 $\mathrm{MHz}$ using conventional pulsed nuclear magnetic resonance techniques. The equilibrium nuclear magnetization $M(\infty)$ is inverted with a $\pi$ pulse. After a time $t$, the nuclear magnetization $M(t)$ is measured by applying a $\pi / 2$ pulse and either (A) sampling the free induction decay or (B) applying a second $\pi$ pulse a time $\tau$ later and sampling the spin echo. These two sequences are denoted $(\pi-t-(\pi / 2))$ and $(\pi-t-(\pi / 2)-\tau-\pi)$ respectively.

In each experiment, $M(\infty)$ was measures by setting $t \sim R_{1}{ }^{-1}$. Then $t$ was set to zero and $M(t)$ was measured at a series of times between zero and $\sim 2.5 \mathrm{~T}_{1}$. Finally, the equilibrium magnetization was measured again and the deviation $M(t)$ from equilibrium was calculated from $M(t)$ on the assumption that the drift in the equilibrium value was linear with time. In all cases, the drift was small. Each series of about 4 experiments was followed by another experiment with the cell evacuated. The signal from this 'vacuum run' was subtracted from all data in the series in order to eliminate possible systematic errors associated with the long term recovery of the receiver-amplifier after the first $\pi$ pulse. In all cases, this 'vacuum correction' was small (e.g., less than $1 \%$ at the $R_{1}$ maximum).

For each individual experiment, the measurements of $M(t)$ could be represented by a single exponential to within the experimental error. The 30 to 40 values of $M(t)$ were fit to [ln $M(t)=-R_{1} t+$ constant] by a least squares routine. The error in $R_{1}$ was determined from the statistical error (Rose 1953) calculated by the least squares procedure and the experimental error 
in measuring the equilibrium magnetization. This latter contribution must be included because of correlations introduced between individual points through the use of a common determination of $M(\infty)$ (Moore and Yalcin 1973). A complete discussion of the error analysis is given in Beckmann (1975).

The spectrometer used is an improved version of that described previously (Beckmann 1971; Beckmann et al. 1972). The RF transmitter is similar to that discussed by Hardy (1966). The recovery time of the current receiver-amplifier system, as measured from the trailing edge of the RF pulses at the transmitter output, is $20 \mu \mathrm{s}$. As in the earlier work, the free induction decay, or spin echo, is sampled by a boxcar integrator (Princeton Applied Research, Model CW-1) and the time $t$ between the first $\pi$ pulse and the $\pi / 2$ pulse is measured by a Hewlett-Packard SL45 counter equipped with a Model 5262A time-interval plug-in. In the current experiment, a digital voltmeter (Monsanto, Model 200A) is used to sample the boxcar output. The outputs of both the voltmeter and the time-interval plugin are recorded digitally on paper tape using a custom interface constructed by Technical Associates Limited (Vancouver). While the signal-to-noise is only moderately improved over that obtained by Beckmann et al. (1972), the new data acquisition system allows systematic computer reduction of large amounts of data, thereby reducing the final errors in $R_{1}$.

The brass sample cell used in the temperature range $77 \mathrm{~K}$ to $273 \mathrm{~K}$ was $8 \mathrm{~cm}$ long with an inside diameter of $2.5 \mathrm{em}$. The 14 turn coil was made of clean, bare copper wire (\#14 gauge); the coil was $4 \mathrm{~cm}$ long with an inside diameter of $1.5 \mathrm{~cm}$. The cell was placed in a small dewar and cooled by a regulated flow of cold nitrogen gas. The temperature was measured by two FD400 diodes which were operated at constant current $(10 \mu \mathrm{A})$ so that the output voltage is a linear function of the temperature (Hardy 1972). ${ }^{8}$ The diodes were placed inside the cell, one at the top and one at the bottom. The gradient, drift, and absolute accuracy of the technique allowed us to measure the temperature to within $1 \mathrm{~K}$. The feed-throughs in the cell for the RF lead and the diode connections were made by passing \#32 gauge brass wire through $0.09 \mathrm{~cm}$ holes and sealing with Stycast Epoxy \#2850FT (\#11 catalyst).

Only one change was made in the room temperature cell described previously (Beckmann et al. 1972); the glass cylinder surrounding the coil was removed. Thus in both sample cells, we had the coil immersed in the sample gas rather than having the gas confined to the interior of the coil. This increases the sample volume, thereby improving the signal-to-noise ratio considerably. However, this design also results in a more inhomogeneous RF field. At densities near and above the $R_{1}$ maximum, it was carefully checked experimentally to ensure that no systematic errors were being made either as a result of diffusion in and out of the coil or as a result of relaxation by paramagnetic impurities on the cell walls. Some systematic effects may be present at densities well below the $R_{1}$ maximum where molecules can diffuse a distance the order of the cell radius in a time $T_{1}$.

The sample gas was research grade methane purchased from Matheson Company Limited. The quoted purity was better than $99.99 \%$. The pressure was measured with a Texas Instruments precision pressure gauge (Model 145) and converted to density using compressibility data (American Petroleum Institute 1957). The error in the density was determined by the uncertainty in the temperature and was always $<1 \%$.

The measured relaxation rates $R_{1}$ are plotted as a function of density $\rho$ in Figs. $3 A, B$, and $C$ for temperatures of 110,150 , and $295 \mathrm{~K}$ respectively. Plots for these three temperatures are

\footnotetext{
${ }^{8}$ Hardy, W. N. 1972. Private communication.
} 
also given in Figs. 3D, E, and $\mathrm{F}$ for $R_{1} \rho$ against $\rho$. The data presented for $295 \mathrm{~K}$ include the measurements of Beckmann et al. (1972). (123 of the 293 room temperature points are new.)

\section{Data Analysis and Interpretation}

In order to obtain insight into the relaxation processes, the relaxation rates $R_{1}$ were calculated with the methods discussed in Sect. 2.C. Since the term in $c_{a} c_{d}$ in [11] is negligible, the theoretical results are parametrized through $\tau_{1}$ and $\tau_{2}$. Each of these is inversely proportional to the density $\rho$

$$
\tau_{i}^{-1}=\langle\sigma v\rangle_{i} \rho, \quad i=1,2
$$

where $v$ and $\sigma$ are respectively, the relative velocity and the cross section of a colliding pair of molecules. The symbol \langle\rangle denotes a canonical ensemble average over an equilibrium ensemble of molecules involved in the type of transition specified by $i$.

In almost all the fits, it was assumed that $\langle\sigma v\rangle_{1}=\langle\sigma v\rangle_{2}=\langle\sigma v\rangle$. The use of the single velocity averaged cross section is equivalent to assuming that all levels broaden equally rapidly with density. It is then convenient to replace the single parameter $\langle\sigma v\rangle$ with the density $\rho \equiv$ $\omega_{0} /\langle\sigma v\rangle$. At this density, there is a maximum (denoted $R$ ) in the relaxation rate due to $H_{\mathrm{SR}} \mathrm{S}$. The value of $\rho$ was determined by fitting the theoretical results to the experimental data at the higher densities where the signal-to-noise ratio was best.

The agreement between theory and experiment is illustrated in Fig. 3. The most striking point is the excellent agreement in the region of the maximum in $R_{1}$; the experimental and theoretical values of $R$ are very close. This fact is particularly significant because $R$ contains no adjustable parameters; that is, within the content of our one-parameter model, $R$ is independent of $\rho$, as can be seen from [5] and [27].

The overall agreement is good at all temperatures, but there are some interesting discrepancies. In evaluating these, it must be remembered that the shape of the curves is important rather than the absolute values. At all three temperatures, the sharpest disagreement occurs at densities below $\rho$ where the theoretical curve is too low. The most probable mechanism is relaxation caused by paramagnetic impurities on the cell walls. The sign of the difference certainly favors this mechanism. In the limit of low densities, the relaxation due to $H_{\mathrm{SR}}$ is proportional to $\rho$. On the other hand, as has been observed recently in ${ }^{3} \mathrm{He}$ (Chapman 1975), the rate due to impurities on the walls is inversely proportional to $\rho$. Thus, at sufficiently low densities, the wall relaxation will dominate and the measured values of $R_{1}$ will exceed those calculated from $H_{\mathrm{SR}}$ alone. The observed difference at low densities cannot be accounted for by increasing the weight assigned to the transitions which do not conserve the distortion energy.

The room temperature data shows two additional discrepancies. From 0.05 to 0.1 amagat, the predicted $R_{1}$ is too large and, above about 1 amagat, the predicted $R_{1} \rho$ increases too rapidly with $\rho$. The suggestion is that our single parameter model weighs too heavily the transitions with large changes in the distortion energy, which are in turn associated with large $J$ values. The $295 \mathrm{~K}$ fit is inferior to those at 110 and $150 \mathrm{~K}$. This is due primarily to the 'centrifugal distortion' transitions. At $295 \mathrm{~K}$, a great many different transitions with $\Delta I$ and/or $\Delta t \neq 0$ occur and none is individually important. In contrast, at the lower temperatures, only a few of these different transitions occur and some are individually significant.

The best values of $\mathrm{p}$ obtained by the fitting procedure are (in amagats):

$$
\begin{aligned}
& \bar{\rho}(110 \mathrm{~K})=(0.0275 \pm 0.0008) \\
& \bar{\rho}(150 \mathrm{~K})=(0.0310 \pm 0.0012) \\
& \bar{\rho}(295 \mathrm{~K})=(0.040 \pm 0.004)
\end{aligned}
$$

The errors have been estimated from the quality of the fits. It is useful to define an effective cross section, $\sigma_{i}$, associated with $\tau_{1}$, by

$$
\bar{\sigma}_{i} \bar{v}=\langle\sigma v\rangle_{i}
$$


where $\mathrm{v}$ is the average relative velocity of a colliding pair of molecules. By setting $\sigma_{1}=\sigma_{2}=\sigma$, an effective cross section for molecular reorientation can be obtained from the values of $\rho$. At $295 \mathrm{~K}, \sigma=19$ $\AA^{2}$, which is about $40 \%$ of the kinetic cross section $\sigma_{\text {KIN }}$ of $46 \AA^{2}$ (Hirschfelder et a/. 1954). The collisions causing spin relaxation in $\mathrm{CH}_{4}$. are therefore intermediate between weak $(\sigma \ll \sigma$ KIN $)$ and strong $\left(\sigma=\sigma_{\mathrm{KIN}}\right)$, a result which has been fairly well established previously (Bloom et al. 1967). From [28], it has been determined that

$$
\bar{\sigma}=\left(2.8 \times 10^{3}\right) T^{-0.88} \AA^{2}
$$

This temperature dependence of $\mathrm{p}$ agrees (Beckmann 1975) with the results obtained by Bloom et al. (1967) at temperatures above $112 \mathrm{~K}$ and densities above the $R_{1}$ maximum.

In order to test the current analysis further, data were taken at $77 \mathrm{~K}$ for densities between 0.0046 and 0.041 amagat. $R_{1}$ was approximately constant at $135 \mathrm{~s}^{-1}$ over this range. By using the values of $\sigma$ predicted by [29], the theoretical rates were calculated. Although the agreement with the measurements is good near the $R_{1}$ maximum, the theoretical values fell below the experimental results by an amount that increased with decreasing density, indicating that some additional mechanism is operative. This behavior is consistent with the onset of wall relaxation, as discussed above.

In an effort to obtain further insight into the relative contributions to $R_{1}$ of different types of transitions, several more complicated methods of parametrizing the theoretical calculation were attempted. These are discussed in Beckmann (1975).

For the data taken here, the relaxation rate can, to good approximation, be written

$$
\text { [30] } R_{1}=R_{1}{ }^{0}\left(c_{\mathrm{a}}\right)+R_{1}{ }^{0}\left(c_{\mathrm{d}}\right)+R_{1}{ }^{1}\left(c_{\mathrm{d}}\right)
$$

Here $R_{1}{ }^{0}\left(c_{\mathrm{a}}\right)$ is the rate due to transitions driven by $H_{\mathrm{SR}} \mathrm{S} \cdot R_{1}{ }^{0}\left(c_{\mathrm{d}}\right)$ and $R_{1}{ }^{1}\left(c_{\mathrm{d}}\right)$ are both rates due to transitions driven by $H_{\mathrm{SR}}^{\mathrm{T}}$; in the former the distortion energy is conserved while in the latter it is not. Using the values of $\rho$ given in [28], these individual terms were calculated for each of the three temperatures studied in detail.

The results for $110 \mathrm{~K}$ are shown in Fig. 4 where various combinations of the different rates multiplied by $\rho$ are plotted against $\rho$ along with the experimental points. $R_{1}{ }^{0}\left(c_{\mathrm{a}}\right)$ is the dominant term. $R_{1}{ }^{1}\left(c_{\mathrm{d}}\right)$ is the same order of magnitude as $R_{1}{ }^{0}\left(c_{\mathrm{d}}\right)$ near $\rho$, but is much larger than $R_{1}{ }^{0}\left(c_{\mathrm{d}}\right)$ at higher densities. The two 'zero frequency' terms $R_{1}{ }^{0}\left(c_{\mathrm{a}}\right)$ and $R_{1}{ }^{0}\left(c_{\mathrm{d}}\right)$ cannot account for the observations; the term $R_{1}{ }^{1}\left(c_{\mathrm{d}}\right)$ makes an important contribution. The terms in which the distortion energy changes must be taken into account.

The importance of $R_{1}{ }^{0}\left(c_{\mathrm{d}}\right)$ and $R_{1}{ }^{1}\left(c_{\mathrm{d}}\right)$ is further demonstrated in Fig. 5, where these individual rates and their sum are plotted against density for $110 \mathrm{~K}$. Also shown are the ratios of the various terms to the total rate at $\rho$ and at the highest density for which measurements were made. These ratios and the shapes of the curves are well-determined by the theory, but the absolute values of the rates contain a large uncertainty. The zero frequency term $R_{1}{ }^{0}\left(c_{\mathrm{d}}\right)$ peaks at $\mathrm{p}$, but the high frequency term $R_{1}{ }^{1}\left(c_{\mathrm{d}}\right)$ does not. If only the single spin conversion transition at $72 \mathrm{MHz}$ for $J=3$ were present, then $R_{1}{ }^{1}\left(c_{\mathrm{d}}\right)$ would peak at 1.2 $\rho$. The contribution of the higher frequency transitions shifts the peak to $1.4 \rho$. While $R_{1}{ }^{0}\left(c_{\mathrm{d}}\right) / R_{1}$ is roughly independent of $\rho, R_{1}{ }^{1}\left(c_{\mathrm{d}}\right) / R 1$ increases significantly with $\rho$. As the temperature increases (with $\rho$ fixed), each of these two ratios decreases. For $R_{1}{ }^{1}\left(c_{\mathrm{d}}\right) / R_{1}$, this decrease occurs primarily because more rotational levels become populated, thereby reducing the importance of any individual transition.

\section{Discussion}

There are two major conclusions we would draw from the work presented here. First, the proton spin relaxation rate $R_{1}$ in $\mathrm{CH}_{4}$ measured at $30 \mathrm{MHz}$ agrees over a wide range in density and temperature with a detailed theory in which the molecular wave functions and energy levels are treated precisely taking into account the permutation symmetry of the four protons, while the collisions are assumed to broaden the rotational energy levels in a uniform manner. The most striking feature in this agreement is the fact that the calculated maximum value of $R_{1}$ fits the experimental data very well even though this maximum 
contains no adjustable parameters. For a given temperature, the shape of the density dependence of $R_{1}$ is very well fit by taking as the single parameter the collision cross section for molecular reorientation i.e. the common broadening parameter of all the rotational levels. The centrifugal distortion transitions make an important contribution to $R_{1}$.

Secondly, the frequency dependence of $R_{1}$ offers the possibility of doing 'relaxation rate spectroscopy' in which the spacing of the distortion energy levels is measured by observing maxima in $R_{1}$ at the corresponding frequencies. Each maximum results from an avoided level crossing which arises when the change in distortion energy matches the change in Zeeman energy. A good candidate for a first study of this type would be the $72 \mathrm{MHz}$ splitting in the $J=3$ state of $\mathrm{CH}_{4}$, corresponding to a magnetic field of approximately $17 \mathrm{kG}$. The experiment may be rather difficult because it must be performed at low densities where the NMR signal is weak. Detailed calculations however, indicate that the $72 \mathrm{MHz}$ splitting should be detectable for $150 \mathrm{~K}$ at a density near $10-2$ amagat with current techniques (Beckmann 1975).

It is instructive to compare the current theory to those discussed in earlier works on relaxation in $\mathrm{CH}_{4}$. This comparison provides both insight into the role played by symmetry considerations and a check on the present work. Dong and Bloom (1970) (referred to hereafter as DB) neglected spin symmetry, treating each proton as being independent. Furthermore, the centrifugal distortion splittings were neglected. They obtained exponential relaxation with

$$
\begin{array}{r}
R_{1}=\frac{2 \pi^{2} k T}{B_{0} h c}\left[c_{\mathrm{a}}{ }^{2} j_{1}\left(\omega_{0}\right)+q\left(\frac{4}{45}\right) c_{\mathrm{d}}{ }^{2} j_{2}\left(\omega_{0}\right)\right] \\
q=1
\end{array}
$$

where $B_{0}$ is in $\mathrm{cm}^{-1}$.

If the present theory is taken to the limit that the distortion splittings vanish, and further, is restricted to the short correlation time limit (i.e. densities well above the $R_{1}$ maximum where $\omega_{0} \tau$ : «1), then an expression similar to [31] is obtained. If these two limits are taken, then it can be shown (Beckmann 1975) that [25] is an excellent approximation to the relaxation equations with

$$
\begin{array}{r}
\boldsymbol{R}=\frac{2 \pi^{2} k T}{\boldsymbol{B}_{0} h c}\left[c_{\mathrm{a}}{ }^{2} j_{1}\left(\omega_{0}\right) \boldsymbol{E}+\left(\frac{4}{45}\right) c_{\mathrm{d}}{ }^{2} j_{2}\left(\omega_{0}\right) S\right] \\
\omega_{0} \tau \ll 1
\end{array}
$$

where

[33] $\boldsymbol{E}=\left(\begin{array}{ll}1 & 0 \\ 0 & 1\end{array}\right)$ and $\boldsymbol{S}=\left(\begin{array}{rr}2 & -5 \\ -1 & 5\end{array}\right)$

The high temperature approximation has been made in arriving at this result. By following the matrix procedure given in Sect. 2.C., we find that the eigenvectors of the relaxation matrix are:

$$
\begin{aligned}
& M_{\alpha}=0.03 M^{(2)}-0.1 M^{(1)} \\
& M_{\beta}=0.97 M^{(2)}+1.1 M^{(1)}
\end{aligned}
$$

The corresponding rate constants $\lambda_{\alpha}$ and $\lambda_{\beta}$ are given by [31] with $q=6$ and 0.8 respectively. Since $\left|M_{\beta}\right| »$ $\left|\mathrm{M}_{\alpha}\right|$, the observed relaxation rate will be very close to $\lambda_{\beta}$, which is in exact agreement with the DB prediction for the $c_{\mathrm{a}}$ term and is in close agreement for the $c_{\mathrm{d}}$ term. Thus, for small distortion splittings and high densities, the effects of spin symmetry are small and may well vanish in a more exact treatment. Some result of this type is to be expected since the spin symmetry quantum numbers and hence spin conversion itself lose their meaning under these conditions.

The agreement between the $c_{\mathrm{a}}$ term in the DB theory and that determined here from [32] can be obtained simply by taking the limit $c_{\mathrm{d}} \rightarrow 0$ and imposing no other restrictions. This agreement acts as a check on the calculations performed in the current work.

McCourt and Hess $(1970,1971)$ treated relaxation in methane under conditions in which the theoretical expressions for the proton relaxation times in $\mathrm{CHD}_{3}$ and $\mathrm{CH}_{4}$ are the same. Their approach is equivalent to treating each proton as independent and can give at best a partial account of the important 
processes since it does not properly treat centrifugal distortion transitions (Ozier 1911). McCourt and Hess (1971) reproduced the DB result given in [31].

Beckmann et al. (1972) (referred to hereafter as BBB) demonstrated that centrifugal distortion transitions are important (see Fig. 2 in BBB) and attempted to fit their data in an empirical manner by introducing the parameter $\Omega \equiv\left|\omega_{\mathrm{k}}-\omega_{0}\right|$ where $\omega_{\mathrm{k}}$ is a single 'average' centrifugal distortion splitting. BBB predicts exponential relaxation with [31] modified so that $q=1$ and $j_{2}\left(\omega_{0}\right)$ is replaced by $j_{2}(\Omega)$. An adequate phenomenological representation of the density dependence of $R_{1}$ was obtained at the single temperature $(295 \mathrm{~K})$ studied. Similar success has been achieved in characterizing measurements in methane-helium mixtures (Lalita 1974). However, aside from the artificial nature, this approach to the distortion transitions has the disadvantage of breaking down near an avoided level crossing, since nonexponential relaxation occurs in that case.

There are two aspects of the present theoretical treatment which should be investigated further. The first involves the form of the nuclear spin relaxation theory. In using rate equations for the populations of the nuclear spin states (see [2]), we have implicitly assumed that the off-diagonal elements of the nuclear spin density matrix do not play an important role. In the modern theory of nuclear spin relaxation, it is customary to include these off-diagonal matrix elements (Abragam 1961) and this has already been done by Hubbard (1958) for the $\mathrm{CH}_{4}$ system. However, Hubbard's theory was formulated for liquids and is not directly applicable to gases at low densities, since Hubbard explicitly neglects the correlation between nuclear spin and molecular rotation that is so important in treating the distortion splittings. Because of this correlation, a complete density matrix treatment would have to include both spin and rotational degrees of freedom. It would appear, a posteriori, from our success in interpreting our experimental data, that these off-diagonal elements of the density matrix are unimportant for the particular system of dilute methane gas. This point merits further consideration, however, since it has not been established on theoretical grounds that they are negligible. The off-diagonal elements may, in fact, be important in interpreting the results of the 'relaxation rate spectroscopy' proposed above. Some conditions under which the off-diagonal elements are unimportant for liquids and solids have been discussed by Emid (1973).

The second aspect of the present treatment which should be investigated further involves the use of the Boltzmann equation for the treatment of the molecular collisions. Procedures for applying such techniques to polyatomic molecules have been described recently by Sanctuary and Snider (1975a, b, c). A proper treatment of molecular collisions may be especially important in the density region where the broadening of pairs of distortion (or rotational) levels becomes comparable with their energy separation.

\section{Acknowledgement}

We wish to thank Dr. W. N. Hardy for some very helpful discussions.

ABRAGAM, A. 1961. The principles of nuclear magnelism (Oxford University Press. London). p. 274. AMERICAN PETROLEUM INSTITUTE. 1957. Research Project 44.

ARMSTRONG, R. L. and COURT N E Y, J. A. 1972. Can. J. Phys. 50. 1262.

BECKMANN. P. A. $\backslash 971$. M.Sc. Thesis. University of British Columbia, Vancouver. B.C. Canada. (Unpublished).

-- 1975. Ph.D. Thesis. University of British Columbia. Vancouver, B.C. Canada. (Unpublished).

BECKMANN. P. A. BLOOM. M. and BURNELL. E. E. 1972. Can. J . Phys. 50. 251.

BLOOM. M. 1972. M.T.P. International reviews of science. ed. by C. A . McDowell (Bulterworth and Co. Ltd.. London).

BLOOM. M. and MORRISON. J. A. 1973. Surface and defect prope1ties of solids. Vol. 2 (The Chemical Society. London). pp. 140- 59.

BLOOM. M. BRIDGES, F .. and HARDY. W. N. 1967. Can. J. Phys. 45, 3533.

CHAPMAN, R. 1975. Phys. Rev. A. 12. 2333.

COURTNEY. J . A. and ARMSTRONG, R. L. 1972. Can. J. Phys. 50, 1252. 
CURL. R. F. 1973. J. Mol. Spectrosc. 48, !65.

CURL. R.F. KASPER. J. V. V. PITZER. K. S. and SATH IANAN DAN. K. 1966. J. Chem. Phys. 44, 4636.

CURL. R. F. KASPER. J.V.Y.. and PITZER. K . S. 1967. J. Chem. Phys. 46. 3220.

DONG. R. Y. and BLOOM. M. 1970. Can. J. Phys. 48. 793.

EMID. S. 1973. Physica. 70. 6 !6.

Fox. K. and OziER. I. ! 970. J . Chern. Phys. 52. 5044.

GORDON. R. G. 1968. Advances in magnetic resonance. Vol. 3. ed. by J. S. Waugh, (Academic Press. New York. NY).

HAMA. J. and MIYAGI. H. 1973. Prog. Theor. Phys. 50, 1142.

HARDY. W. N. 1966. Can. J . Phys. 44.265.

HERZBERG, G. 1969. Proceeding of the Colloquium on High Resolution Molecular Spectroscopy.

Dijon. France. (Unpublished).

-- 1971. Highlights of astronomy. ed. by C. DeJager (Reidel. Dordrecht).

HIRSCHFELDER. J. O. CURTISS C. F., and BIRD. R.B. 1954. Molecular theory of gases and liquids (J. Wiley and Sons Inc. New York. NY).

HoLT, C. W., GERRY. M. C. L., and OZIER, I. 1975. Can. J. Phys. 53. 1971.

H U BBARD, P. S. !958. Phys. Rev. 109. 1 ! 53.

JAHN, H.A. 1938. Proc. R. Soc. Lond. A . 168. 469.

LALITA . K. 1974. Can. J. Phys. 52, 876.

McCoURT, F. R . and HESS. S., 1970. Z. Naturforsch. 25a, 1169.

--1971. Z. Naturforsch. 26a. 1234.

MOORE. W. S. and YALCIN, T. 1973. J. Magn. Reson. II. 50.

OZIER. I. 197 1. Z. Naturforsch. 26a, 1232.

-- 1974. J. Mol. Spectrosc. 53.336.

OZIER. I. and FOX, K. 1970. J. Chern. Phys. 52. 1416.

OZIER. I. and ROSENBERG. A. 1973. Can. J. Phys. 51. 1882.

OZIER, I. and YI. P. N . !967. J. Chern. Phys. 47. 5458.

OZIER, I., CRAPO. L M . . and LEE. S. S. !968. Phys. Rev. 172. 63.

OZIER, I., Y1. P. N., KHOSLA, A., and RAMSEY, N. F. 1970. Phys. Rev. Lett. 24. 642.

PRESS. W. and KOLLMAR. A. 1975. Solid State Commun. 17. 405.

ROSE. M. E. 1953. Phys. Rev . $91.6 \backslash 0$.

SANCTUARY. B . C. and S N I D E R , R . F . 1975a,b,c. Can. J. Phys. 53. 707. 723. 739.

TARRAGO. G., DANG-NHU, M., POUSSIGUE. G., GUELACHVILI. G., and AMIOT, C. 1975. J. Mol. Spectrosc. 57. 246.

YI. P., OZIER. I., and ANDERSON. C. H. 1968. Phys. Rev. 165. 92.

YI, P., OZiER. I., and RAMSEY. N. F. 1971. J Chem. Phys. 55. 5215.

\section{Appendix: The Spin-Rotation Matrix Element}

The spin rotation interaction $H_{\mathrm{SR}}$ provides the coupling between the spin and rotational degrees of freedom that is essential to the relaxation process; its matrix elements determine the transition rates as specified by [3] and [4]. Aside from their importance in the present work, these matrix elements may be useful in a variety of other problems such as the extension of the current treatment of spin relaxation, studies of avoided crossings by molecular beam methods, and calculation of second-order shifts in the nuclear hyperfine energies. We therefore list the matrix elements here explicitly: 
[A.1 $\left\langle H_{\mathrm{SR}}{ }^{\mathrm{S}}\left(\gamma^{\prime}, \gamma\right)\right\rangle=-\sqrt{2} h c_{\mathrm{a}} \delta\left(\gamma^{\prime}, \gamma\right) \delta\left(t^{\prime}, t\right)[\delta(\gamma, F)+\sqrt{3} \delta(\gamma, A)][J(J+1)]^{1 / 2} L_{J}\left(I m m^{\prime} m^{\prime}{ }_{j} ; I m m_{J}\right)$

[A.2] $\left\langle H_{\mathrm{SR}^{\top}}{ }^{\top}\left(\gamma^{\prime}, \gamma\right)\right\rangle=-(1 / 3) h c_{\mathrm{d}} \delta(\gamma, F)[J(J+1)]^{-1 / 2} M_{J_{\mathrm{p}}}\left(\gamma^{\prime} t^{\prime} ; F t\right) L_{J}\left(I m^{\prime} m_{J}^{\prime} ; 1 m m_{J}\right)$

where

[A.3]

$$
\begin{aligned}
L_{J}\left(I^{\prime} m^{\prime} m^{\prime}{ }_{J} ; I m m_{j}\right) & =\sum_{\nu}(-1)^{v} C\left(I, 1, I^{\prime} ; m, v_{,}, m^{\prime}\right) C\left(J, 1, J ; m_{J},-v_{,} m^{\prime}{ }_{J}\right) \\
M_{J \rho}\left(F t^{\prime} ; F t\right) & =\sqrt{2} S_{J_{\rho}}\left(F x t^{\prime} ; F z t\right) \\
M_{J_{\rho}}\left(A t^{\prime} ; F t\right) & =(-1)^{J+\rho}(\sqrt{6} / 2) S_{J \rho}\left(A t^{\prime} ; F y t\right) \\
M_{J \rho}\left(E t^{\prime} ; F t\right) & =(-1)^{J+1} 2[\delta(\rho, 1)+\sqrt{3} \delta(\rho, 2)] S_{J \rho}\left(E 1 t^{\prime} ; F y t\right)
\end{aligned}
$$

and

$$
S_{J_{\rho}}\left(\gamma^{\prime} \xi^{\prime} t^{\prime} ; \gamma \xi t\right)=\sum_{K^{\prime} K^{\prime}} A_{t^{\prime} K^{\prime}}\left(J \gamma^{\prime} \xi^{\prime} \rho\right) A_{t K}(J \gamma \xi \rho) Q_{J}\left(K^{\prime} K\right)
$$

[A.6] $Q_{J}\left(K^{\prime} K\right)=\left\{\left[1+\delta\left(K^{\prime}, 0\right)\right][1+\delta(K, 0)]\right\}^{-1 / 2}$

$$
\begin{aligned}
& \times\left\{(1+2 K)[(J+K+1)(J-K)]^{1 / 2} \delta\left(K^{\prime}, K+1\right)\right. \\
& \left.-(1-2 K)[(J-K+1)(J+K)]^{1 / 2}\left[\delta\left(K^{\prime}, K-1\right)+(-1)^{\prime} \delta\left(K^{\prime}, 1-K\right)\right]\right\}
\end{aligned}
$$

$\left\langle H_{\mathrm{SR}} \mathrm{S}\left(\gamma^{\prime}, \gamma\right)\right\rangle$ has been calculated earlier (e.g., Ozier et al. (1968)), and is included here only to avoid ambiguities arising from differences in notation. $\left\langle H_{\mathrm{SR}}{ }^{\mathrm{T}}\left(\gamma^{\prime}, \gamma\right)\right\rangle$ has been calculated previously only for cases diagonal in $\gamma$ and $t$ (Ozier et al. 1968) and for the special case of $J=2, \gamma^{\prime}=E, \gamma=F$ (e.g., Curl (1973)). The general expressions given here agree with these earlier results. 
Tables:

TABLE 1. Summary of transition probability functions

\begin{tabular}{lccc}
\hline Function & $\begin{array}{c}\text { Coupling } \\
\text { constant }\end{array}$ & $I^{\prime} \leftrightarrow I$ & $\begin{array}{c}\text { Does the } \\
\text { centrifugaldistortion } \\
\text { energy change? }\end{array}$ \\
\hline$\hat{A}(J A \rho)$ & $c_{\mathrm{a}}{ }^{2}$ & $2 \leftrightarrow 2$ & no \\
$\hat{A}(J F \rho)$ & $c_{\mathrm{a}}{ }^{2}$ & $1 \leftrightarrow 1$ & no \\
$\hat{B}(J F \rho)$ & $c_{\mathrm{a}} c_{\mathrm{d}}$ & $1 \leftrightarrow 1$ & no \\
$\hat{D}(J F \rho)$ & $c_{\mathrm{d}}{ }^{2}$ & $1 \leftrightarrow 1$ & no \\
$\hat{E}_{m m} \cdot(J F \rho)$ & $c_{\mathrm{d}}{ }^{2}$ & $1 \leftrightarrow 1$ & yes \\
$\hat{E}_{n m m^{\prime}}(J A \rho)$ & $c_{\mathrm{d}^{2}}{ }^{2}$ & $2 \leftrightarrow 1$ & yes \\
$\hat{E}_{m 0}(J E \rho)$ & $c_{\mathrm{d}}{ }^{2}$ & $0 \leftrightarrow 1$ & yes \\
\hline
\end{tabular}

TABLE 2. Functions $R^{t}\left(I^{\prime} m^{\prime}\right)^{a}$

\begin{tabular}{lrrl}
\hline$I$ & $I^{\prime}$ & \multicolumn{1}{c}{$m^{\prime}$} & \multicolumn{1}{c}{$R^{\prime}\left(I^{\prime} m^{\prime}\right)$} \\
\hline 2 & 2 & 2 & $-\frac{1}{2} W(2221)-W(2211)$ \\
2 & 2 & 1 & $-\frac{1}{2} W(2221)-W(2111)-W(2110)$ \\
2 & 2 & 0 & 0 \\
2 & 2 & -1 & $-\frac{1}{2} W(2221)-W(2111)-W(2-110)$ \\
2 & 2 & -2 & $-\frac{1}{2} W(2221)-W(2-21-1)$ \\
2 & 1 & 1 & $2 W(2211)+W(2111)$ \\
2 & 1 & 0 & $W(2110)-W(2-110)$ \\
2 & 1 & -1 & $2 W(2-21-1)+W(2111)$ \\
1 & 2 & 2 & $\frac{1}{2} W(2211)$ \\
1 & 2 & 1 & $W(2111)$ \\
1 & 2 & 0 & $W(2011)-W(201-1)$ \\
1 & 2 & -1 & $W(2111)$ \\
1 & 2 & -2 & $\frac{1}{2} W(2-21-1)$ \\
1 & 1 & 1 & $-W(1110)-W(2211)-W(2111)-W(2011)-W(0011)$ \\
1 & 1 & 0 & $W(1011)-W(101-1)$ \\
1 & 1 & -1 & $-W(1-110)-W(2-21-1)-W(2111)-W(201-1)-W(001-1)$ \\
1 & 0 & 0 & $W(0011)-W(001-1)$ \\
\hline${ }^{\circ}$ The simplification used in constructing the table from [22] is given on p. I17 of Beckmann (1975).
\end{tabular}




\section{Figures:}

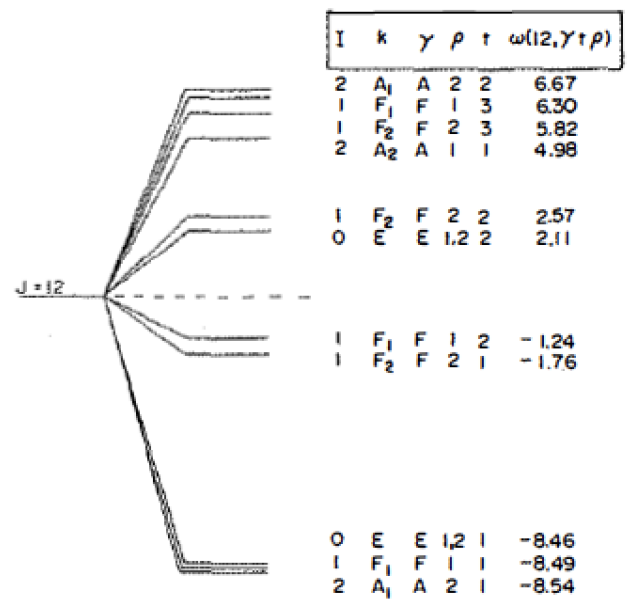

Fig. 1. The rotational energy level diagram for the $J=12$ state of $\mathrm{CH}_{4}$. The reference energy is determined by $H_{\mathrm{ROT}}{ }^{\mathrm{s}}$ introduced in [6]. The splittings $\omega(J \gamma t \rho)$ shown here for $J=12$ are the eigenvalues of the operator $D_{\mathrm{r}} \Omega_{4}$ with $D_{\mathrm{T}}$ taken from Holt et al. (1975). The diagram is drawn to scale. The quantum numbers $(\gamma t \rho)$ and $k$ are defined in Sect. 2.

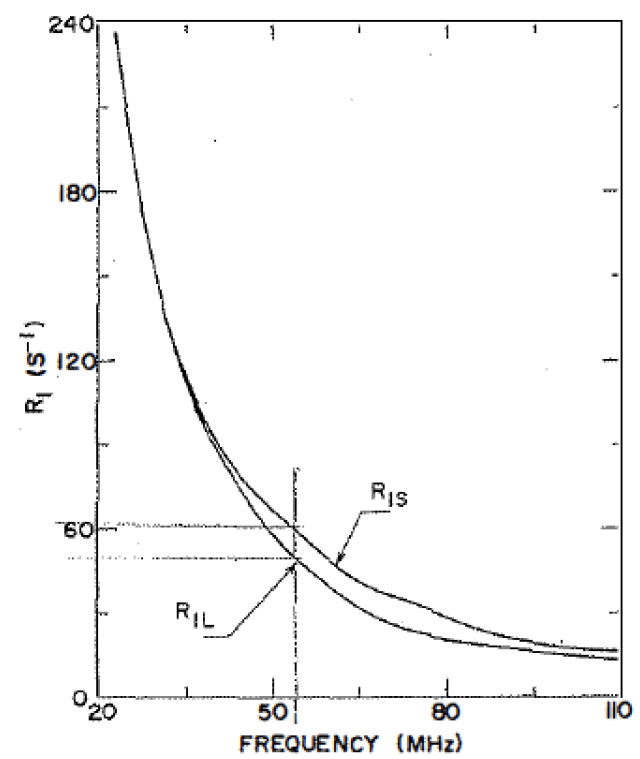

FIG. 2. The dependence on the nuclear Larmor frequency of the relaxation rates $R_{15}$ and $R_{1 \mathrm{LL}}$ which characterize the short and long time behaviour respectively, of $M(t)$. See Sect. 2.C. for detailed definitions of these rates. The calculation was done for a temperature of $150 \mathrm{~K}$ and a density of 0.01 amagats with the iterative procedure based on [20]. Notice the maxima in $\left[R_{1 \mathrm{~S}}-R_{1 \mathrm{~L}}\right]$ at 55 and $76 \mathrm{MHz}$. 

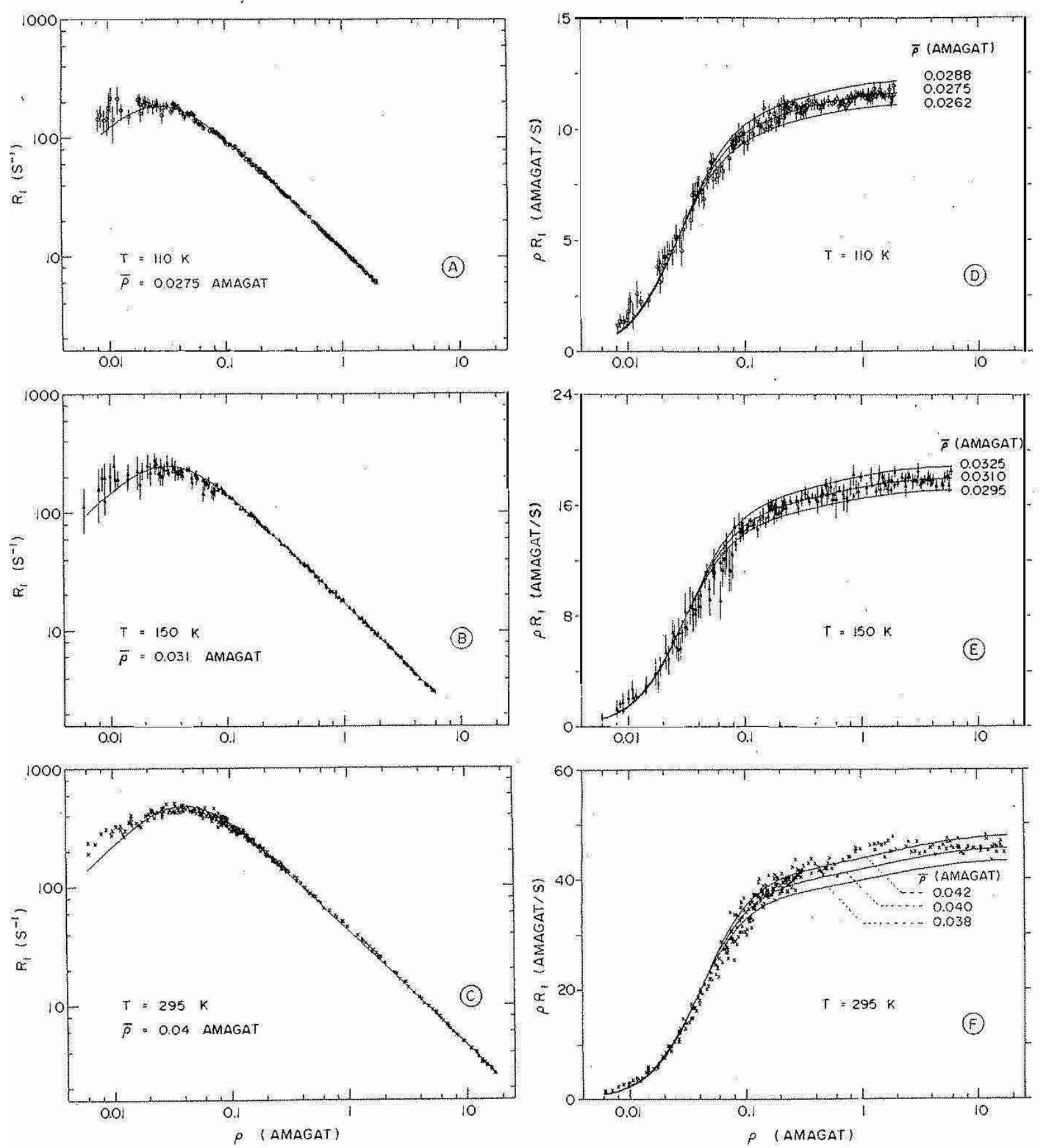

Fic. 3. The dependence on density $\rho$ of the relaxation rate $R_{1}$ for $(A) 110 \mathrm{~K},(B) 150 \mathrm{~K},(C) 295 \mathrm{~K}$; and of $\rho R_{1}$ for $(D) 110 \mathrm{~K},(E) 150 \mathrm{~K}$, and $(F) 295 \mathrm{~K}$. The points represent the experimental data. $58 \%$ of the points at $295 \mathrm{~K}$ were taken from Beckmann et al. (1972). The experimental errors are shown for 110 and $150 \mathrm{~K}$, but not for $295 \mathrm{~K}$. The theoretical results are represented by the solid lines; in each case, the value of $\bar{\rho}$ is indicated. For $(A),(B)$, and $(C)$, only the best fit is shown; for $(D)$, $(E)$, and $(F)$, curves are drawn for the best fit value of $\bar{\rho}$ and for values of $\bar{\rho}$ which are $5 \%$ higher and $5 \%$ lower. 


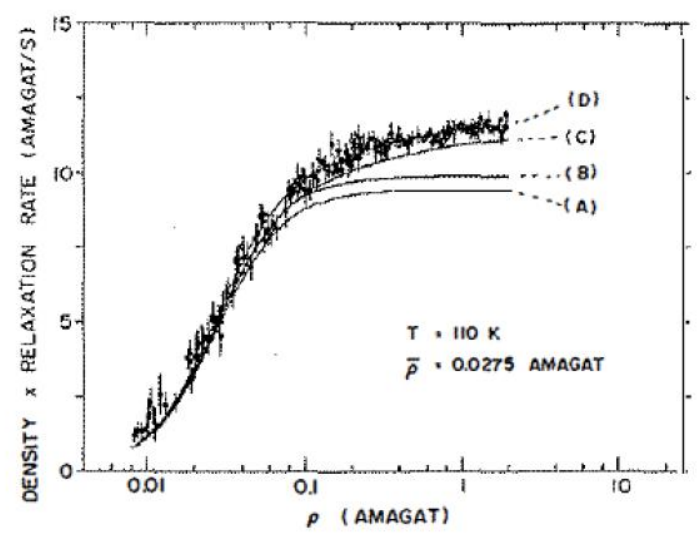

Fig. 4. Different combinations of the three contributions to $\rho R_{1}$ plotted in solid lines as a function of $\rho$ for $110 \mathrm{~K}:(A) \rho R_{1}{ }^{\circ}\left(c_{\mathrm{a}}\right),(B)\left[\rho R_{1}{ }^{\circ}\left(c_{\mathrm{a}}\right)+\rho R_{1}{ }^{\circ}\left(c_{\mathrm{d}}\right)\right],(C)$ $\left[\rho R_{1}{ }^{\circ}\left(c_{\mathrm{a}}\right)+\rho R_{1}{ }^{1}\left(c_{\mathrm{d}}\right)\right]$, and $(D)$ the total $\rho R_{1}$. The different relaxation rates are defined in connection with [30]. The curves are calculated for the best fit value of $\bar{\rho}$ shown. The points are the experimental data.

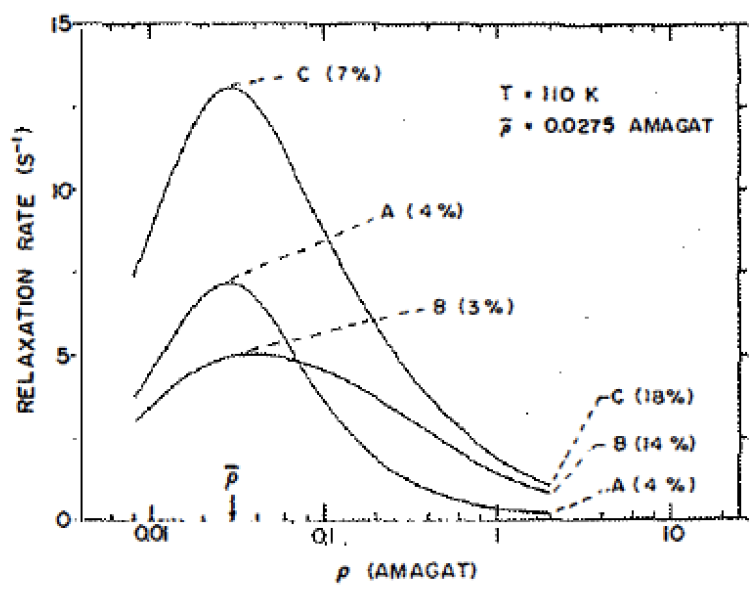

FIG. 5. Different contributions of the tensor spin rotation interaction to the total relaxation rate $R_{1}$ plotted as a function of $\rho$ for $110 \mathrm{~K}:(A) R_{1}{ }^{0}\left(c_{\mathrm{d}}\right),(B) R_{1}{ }^{1}\left(c_{\mathrm{d}}\right)$, and (C) the sum $\left[R_{1}{ }^{\circ}\left(c_{\mathrm{d}}\right)+R_{1}{ }^{1}\left(c_{\mathrm{d}}\right)\right] . R_{1}{ }^{\circ}\left(c_{\mathrm{d}}\right)$ conserves the centrifugal distortion energy and $R_{\mathrm{L}}{ }^{1}\left(c_{\mathrm{d}}\right)$ does not; see [30]. The curves are calculated for the best fit value of $\bar{\rho}$ specified; this value of $\bar{\rho}$ is plotted on the $\rho$ axis. For the densities shown, there is indicated on each curve the ratio of the corresponding relaxation rate to the total. These ratios and the shapes of the curves are well determined, but the absolute values of the rates contain a large uncertainty. 